\title{
Facing the coronavirus pandemic era
}

\author{
Emanuela Brusadelli, ${ }^{1}$ Christian Schubert, ${ }^{2}$ Silvia Salcuni ${ }^{3}$ \\ ${ }^{1}$ School of Psychology, Faculty of Arts, Social Sciences \& Humanities, University of Wollongong, Australia; ${ }^{2}$ Univ.-Klinik für Medi- \\ zinische Psychologie, Medizinische Universität, Innsbruck, Germany; ${ }^{3}$ Department of Developmental and Socialization Psychology, \\ University of Padova, Italy
}

The year 2020 has been defined by the emergency of the spread of the coronavirus 2019 (COVID-19) pandemic, with extraordinary repercussions for people's everyday lives across the world.

This unprecedented large-scale event is likely to overtake the effect of previous infectious disease outbreaks, such as severe acute respiratory syndrome (Wilder-Smith, Chiew \& Lee, 2020).

The COVID-19 crisis has unfolded with extreme speed and has resulted in a high degree of uncertainty requiring people to deal with measures to prevent the diffusion of this invisible enemy, such as lockdown and social isolation, as well as with macroeconomic consequences for families and whole nations. In addition to the severe impact on people's physical health, the psychological impact of COVID-19 is undeniable, further complicated by the socioeconomic effects that are likely to be profound and long lasting (Rettie \& Daniels, 2020).

Research in Psychotherapy: Psychopathology, Process and Outcome seeks to contribute to the understanding of this worldwide phenomenon with a special issue featuring international contributions with various approaches, focuses and methodologies. Taken together,

Correspondence: Emanuela Brusadelli, School of Psychology, Faculty of Arts, Social Sciences \& Humanities, University of Wollongong NSW 2522 Australia.

E-mail: emanuela@uow.edu.au

Citation: Brusadelli, E., Schubert, C., \& Salcuni, S. (2020). Facing the coronavirus pandemic era. Research in Psychotherapy: Psychopathology, Process and Outcome, 23(3), 199-201. doi: 10.4081/ripppo.2020.509

Received for publication: 4 December 2020.

Accepted for publication: 4 December 2020.

This work is licensed under a Creative Commons Attribution NonCommercial 4.0 License (CC BY-NC 4.0).

${ }^{\circ}$ Copyright: the Author(s), 2020

Licensee PAGEPress, Italy

Research in Psychotherapy:

Psychopathology, Process and Outcome 2020; 23:199-201

doi:10.4081/ripppo.2020.509 the following papers investigate the enduring consequences on mental health and provide insights into effective psychological interventions that can help people deal with psychological distress and prevent further psychopathological effects.

The issue opens with a paper based on the psychoanalytic notion of patient-therapist intersubjectivity. Knight (2020a), in 'Living under lockdown in the shadow of the COVID-19 pandemic in South Africa: anxious voices from the unplanned shift to online therapy', presents a narrative, phenomenological description of the experiences of some of her South African patients during the lockdown. It discusses the shift to online therapy and includes the author's experiences and thoughts about therapeutic engagement.

The ensuing series of papers focuses on the psychological consequences of the pandemic - in terms of symptoms, protective factors and mediators - on both community and clinical samples. Gullo, Misici, Teti, Liuzzi, and Chiara (2020) present 'Going through the lockdown: a longitudinal study on the psychological consequences of the coronavirus pandemic', which explores the effects of the lockdown at four time points, then also looking at postlockdown anxiety. Their results indicate higher levels of depression and anxiety and lower levels of coping and perceived social support in comparison with normative samples for each of the four time points.

From a similar perspective, the paper by Faustino, Vasco, Delgado, Farinha, and Guerreiro (2020), titled ' $E x$ ploring the Impacts of COVID-19 related social distancing on loneliness, psychological needs and symptomatology', explores the relationships between loneliness, symptomatology and the regulation of psychological needs. The results underline that loneliness is positively correlated with symptomatology and difficulties in the regulation of psychological needs. The authors also show how these relationships are mediated by psychological distress and psychological well-being.

In search of protective factors, Rotarescu et al. (2020) evaluate in 'How anxious did you feel during lockdown? The roles resilience, living environment, and gender play on the level of anxiety state during pandemic isolation' how factors such as resilience, gender, urban/rural environment and pre-existing anxiety can impact $\mathrm{ON}$ anxious states states due to forced home isolation. Their results indicate 
that people living in small urban areas have a higher level of anxiety, and that experiential avoidance, anxiety trait and resilience play a significant role in the level of anxiety state. Gender, however, was found to have no moderating role in the relationship between resilience and anxiety states.

From a similar perspective, the paper by Kim and Crimmins (2020), 'Age differences in the relationship between threatening and coping mechanisms and preventive behaviors in the time of COVID-19 in the United States: Protection Motivation Theory', investigates how four cognitive mediators affected the adoption of protective activities in younger and older adults during the beginning of the COVID-19 pandemic. The results show some differences between these two groups that should be considered when developing behavioral modifications.

The paper by Bonazza et al. (2020), 'Psychological outcomes after hospitalization for COVID-19: data from a multidisciplinary follow-up screening program for recovered patients', focuses on the psychological condition of previously hospitalized COVID-19 patients two months after their discharge. The recovered COVID-19 patients reported negative consequences on their mental health. In particular, impaired outcomes were associated with female gender, while age was negatively correlated with anxiety symptoms.

Considering new technologies in psychological interventions, Reis, Matthews, and Grenyer (2020), in their contribution 'Characteristics of effective online psychotherapy for personality disorder in adolescents: Implications for the COVID-19 global pandemic', investigate available online programs specific to personality disorders in adolescence; their paper also reviews the literature on online psychotherapy options for adolescents with personality disorders. Taken together, the contribution underlines the urgency of reducing the burden and impact of personality disorder in adolescents and illustrates several strategies for the development and implementation of novel online treatments.

In their paper, 'Online Supportive Group as social intervention to face COVID lockdown. A qualitative study on psychotherapists, psychology trainees and students, and community people', Brusadelli et al. (2020) apply the Linguistic Inquiry and Word Count to analyze data from focus groups following a supportive psychoanalytically-oriented intervention provided to psychologists in training, psychology students, psychotherapists and the general population. Their results on perceptions of the intervention and of the pandemic show that social role was associated with different ways of experiencing and sharing emotions.

The paper concluding this issue adopts a countertransference perspective: Knight (2020b), in 'My story in response to the stories of my patients' experience of the impact of the COVID-19 pandemic: a relational psychoanalytic approach', documents her story in response to three patients' experiences on the impact of the COVID19 pandemic.
We thank all the authors and reviewers for their thorough and valuable collaboration. It was through their work that this special issue, born in such a difficult and particular period of history, could be realized. We may all be proud of the drive of our creativity, our clinical spirit and our scientific commitment, which have led to creative collaboration and strong relationships.

\section{References}

Bonazza, F., Borghi, L., Cao di San Marco, E., Piscopo, K., Bai, F., d'Arminio Monforte, A. \& Vegni, E. (2020). Psychological outcomes after hospitalization for COVID-19: data from a multidisciplinary follow-up screening program for recovered patients. Research in Psychotherapy: Psychopathology, Process and Outcome, 23(3), 247-255.

Brusadelli, E., Ferrari, L., Benetti, M., Bruzzese, S., Tonelli, G. M., \& Gullo, S. (2020). Online Supportive Group as social intervention to face COVID lockdown. A qualitative study on psychotherapists, psychology trainees and students, and community people. Research in Psychotherapy: Psychopathology, Process and Outcome, 23(3), 279-290.

Faustino, B., Vasco, A. B., Delgado, J., Farinha, A., \& Guerreiro, J. C. (2020). Exploring the impacts of COVID-19 related social distancing on loneliness, psychological needs and symptomatology. Research in Psychotherapy: Psychopathology, Process and Outcome, 23(3), 222-230.

Gullo, S., Misici, I., Teti, A., Liuzzi, M. \& Chiara, E. (2020). Going through the lockdown: a longitudinal study on the psychological consequences of the coronavirus pandemic. Research in Psychotherapy: Psychopathology, Process and Outcome, 23(3), 211-221.

Kim, J. K., \& Crimmins, E. (2020). Age differences in the relationship between threatening and coping mechanisms and preventive behaviors in the time of COVID-19 in the United States: Protection Motivation Theory. Research in Psychotherapy: Psychopathology, Process and Outcome, 23(3), 239-246.

Reis, S., Matthews, E., \& Grenyer, B. F. S. (2020). Characteristics of effective online psychotherapy for personality disorder in adolescents: implications for the COVID-19 global pandemic. Research in Psychotherapy: Psychopathology, Process and Outcome, 23(3), 256-278.

Rettie, H., \& Daniels, J. (2020). Coping and tolerance of uncertainty: predictors and mediators of mental health during the COVID-19 pandemic. American Psychologist. [Advance online publication: http://dx.doi.org/10.1037/amp $0000710]$.

Rotarescu, V.S. Nedelescu, B., Nedelea, D., Neagu, A., Otesanu, G. A., Mirescu, A., Matei, D. B. Mircea, I. A., Necsulescu, A. G., \& Tudor, L. C. (2020). How anxious did you feel during lockdown? The roles resilience, living environment, and gender play on the level of anxiety state during pandemic isolation. Research in Psychotherapy: Psychopathology, Process and Outcome, 23(3), 231-238.

Wilder-Smith, A., Chiew, C. J., \& Lee, V. J. (2020). Can we contain the COVID-19 outbreak with the same measures as for SARS? The Lancet Infectious Diseases, 20(5), e102e107.

Knight, Z. G. (2020a). Living under lockdown in the shadow of the COVID-19 pandemic in South Africa: anxious voices 
from the unplanned shift to online therapy. Research in Psychotherapy: Psychopathology, Process and Outcome, 23(3), 202-210.

Knight, Z. G. (2020b). My story in response to the stories of my patients' experience of the impact of the COVID-19 pandemic: a relational psychoanalytic approach. Research in Psychotherapy: Psychopathology, Process and Outcome, 23(3), 291-297. 